\title{
Development of novel echogenic-imageable thermosensitive liposome for optimizing tumor drug distribution using ultrasound guided HIFU
}

\author{
Ashish Ranjan*, Danny Maples, Ryan Newhardt, Venkatesan Perumal \\ From Current and Future Applications of Focused Ultrasound 2014. 4th International Symposium \\ Washington, D.C, USA. 12-16 October 2014
}

\section{Background/introduction}

A major challenge in HIFU mediated Image Guided Drug Delivery (IGDD) is developing accurate means to implement real-time drug delivery control, and to optimize intratumoral drug distribution. To facilitate clinical translation, objectives of this study were to: 1 ) develop echogenic E-LTSL, a low temperature sensitive liposome co-loaded with an US contrast agent (Perfluoropentane, PFP) and doxorubicin, 2) determine stability of contrast agent encapsulation and characterize doxorubicin release from E-LTSL and 3) investigate the ability of E-LTSL to report on realtime doxorubicin distribution with Ultrasound (US)-guided hyperthermia.

\section{Methods}

E-LTSL was loaded passively with PFP using an innovative 1-step sonoporation method and actively loaded with doxorubicin. Doxorubicin release and PFP imageability from E-LTSL in phantoms was quantified by fluorescence spectroscopy, ultrasound imaging and transmission electron microscopy (TEM) in combination with mild hyperthermia $\left(40-42^{\circ} \mathrm{C}\right)$. Ultrasound imageability of E-LTSL was determined in vivo in a mouse xenograft model of human prostate cancer.

\section{Results and conclusions}

TEM images confirmed that the PFP emulsion formation is contained within LTSL. Phantom study clearly showed that only E-LTSLs are echogenic. Temperature $v s$. size increase and drug release kinetics of E-LTSL demonstrated no difference with control. Doxorubicin release in physiological buffer was $<5 \%$ in $1 \mathrm{hr}$ at baseline $\left(25^{\circ} \mathrm{C}\right)$ and body temperatures $\left(37^{\circ} \mathrm{C}\right)$, vs. $>99 \%$ release with hyperthermia $\left(\sim 41^{\circ} \mathrm{C}\right)$. Intensity of observed ultrasound image with respect to temperature in the range of $31-40^{\circ}$ C correlated strongly to the formation of gas bubbles in E-LTSL, and stabilized to a fixed intensity at the transition temperature. After the transition temperature of E-LTSL reached, the US intensity increased again similar to Dox release. Synthesized E-LTSLs were imageable in vivo and were stable in aqueous environment, with no visual evidence of particle aggregation after $48 \mathrm{hr}$ storage at $4{ }^{\circ} \mathrm{C}$. In conclusion, an US imageable heat sensitive liposome formulation co-loaded with doxorubicin and an US contrast agent was developed. Stability, imageability, and US monitoring of contrast agent and Dox release suggest that US-guided drug delivery from E-LTSL may assist physicians in real-time tumor drug delivery mapping. In vivo US-guided HIFU in combination with E-LTSL to demonstrate enhanced tumor drug distribution in $\mathrm{C} 26$ colon cancer model is currently in progress. This technology has potential for clinical translation.

\section{Acknowledgements (Funding)}

Research reported in this publication was supported by the National Cancer Institute of the National Institutes of Health under Award Number R15CA179369, and Oklahoma Center for the Advancement of Science and Technology (OCAST) HR13-217. The content is solely the responsibility of the authors and does not necessarily represent the official views of the National Institutes of Health.

Published: 30 June 2015

\section{doi:10.1186/2050-5736-3-S1-P73}

Cite this article as: Ranjan et al: Development of novel echogenicimageable thermosensitive liposome for optimizing tumor drug distribution using ultrasound guided HIFU. Journal of Therapeutic Ultrasound 2015 3(Suppl 1):P73. 